Research Paper

\title{
Target Therapy With Vaccinia Virus Harboring IL-24 For Human Breast Cancer
}

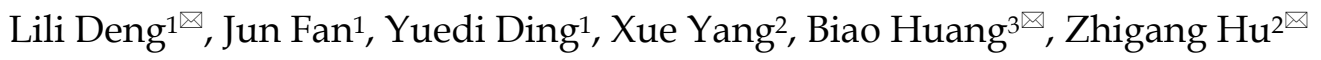 \\ 1. NHC Key Laboratory of Nuclear Medicine, Jiangsu Key Laboratory of Molecular Nuclear Medicine, Jiangsu Institute of Nuclear Medicine, Wuxi 214063, \\ China. \\ 2. Wuxi Children's Hospital, Wuxi People's Hospital affiliated to Nanjing Medical University, Wuxi 214023, China. \\ 3. School of Life Science, Zhejiang Sci-Tech University, Hangzhou 310018, China. \\ $\triangle$ Corresponding author: Lili Deng, denglili@jsinm.org; Jiangsu Institute of Nuclear Medicine, QianRong Road \#20, Wuxi, Jiangsu 214063, China. Tel: \\ +(86)051085508776; Biao Huang, huangbiao@jsinm.org; Zhejiang Sci-Tech University, Xiasha District, Street 2, \#926, Hangzhou, Zhejiang 310018, China. Tel: \\ +(86)057186843187; Zhigang Hu, jswxhzg@163.com; Wuxi Children's Hospital, Wuxi People's Hospital affiliated to Nanjing Medical University, Qingyang Road \\ \#299-1, Wuxi, Jiangsu 214023, China. Tel: +(86)051085350227.
}

(C) The author(s). This is an open access article distributed under the terms of the Creative Commons Attribution License (https://creativecommons.org/licenses/by/4.0/). See http://ivyspring.com/terms for full terms and conditions.

Received: 2019.06.14; Accepted: 2019.10.26; Published: 2020.01.01

\begin{abstract}
Background: Breast cancer is a heterogeneous disease with high aggression and novel targeted therapeutic strategies are required. Oncolytic vaccinia virus is an attractive candidate for cancer treatment due to its tumor cell-specific replication causing lysis of tumor cells as well as a delivery vector to overexpress therapeutic transgenes. Interleukin-24 (IL-24) is a novel tumor suppressor cytokine that selectively induces apoptosis in a wide variety of tumor types, including breast cancer. In this study, we used vaccinia virus as a delivery vector to express IL-24 gene and antitumor effects were evaluated both in vitro and in vivo. Methods: The vaccinia virus strain Guang9 armed with IL-24 gene (VG9-IL-24) was constructed via disruption of the viral thymidine kinase (TK) gene region. The cytotoxicity of VG9-IL-24 in various breast cancer cell lines was assessed by MTT and cell cycle progression and apoptosis were examined by flow cytometry. In vivo antitumor effects were further observed in MDA-MB-231 xenograft mouse model. Results: In vitro, VG9-IL-24 efficiently infected and selectively killed breast cancer cells with no strong cytotoxicity to normal cells. VG9-IL-24 induced increased number of apoptotic cells and blocked breast cancer cells in the G2/M phase of the cell cycle. Western blotting results indicated that VG9-IL-24-mediated apoptosis was related to $\mathrm{PI} 3 \mathrm{~K} / \beta$-catenin signaling pathway. In vivo, VG9-IL-24 delayed tumor growth and improved survival. Conclusions: Our findings provided documentation that VG9-IL-24 was targeted in vitro and exhibited enhanced antitumor effects, and it may be an innovative therapy for breast cancer.
\end{abstract}

Key words: oncolytic vaccinia virus; interleukin-24; breast cancer; gene therapy; apoptosis.

\section{Introduction}

Breast cancer is the principal cause of cancer-related death in women. Despite the development in early diagnostics and therapies, the overall survival rates remain unchanged during last two decades [1]. Specially, triple-negative breast cancer (TNBC), an aggressive subtype of breast cancer with poorest prognosis and lower survival, is refractory to current therapies duo to lack expression of estrogen receptor (ER), progesterone receptor (PR) and the overexpression of the HER2/neu receptor. Traditional therapies for breast cancer, such as surgery, chemotherapy and radiotherapy, have a series of adverse effects including inefficient curative effects, cognitive impairments, tumor metastasis increase and resistance to established therapies. Therefore, novel therapeutic strategies for breast cancer therapy are strongly required.

Oncolytic vaccinia virus represents a potential strategy for cancer therapy due to its appealing features such as extensive safety as a live vaccine and efficient delivery to metastatic tumors [2-4]. Besides, oncolytic vaccinia virus can also be used as gene 
expression carrier due to its large size and expression using its own enzyme systems. With the replication of vaccinia virus, the copies of carried genes are also increased, leading to efficient expression levels in tumor tissues. So far, vaccinia virus has been modified to carry various antigens, cytokines, and immunostimulatory molecules, such as Wyeth strain modified by insertion of granulocyte microphage colony-stimulating (GM-CSF) [5], and Western Reserve strain armed with interleukin (IL)-12[6], IL-4 [7], IL-10 [8] and IFN- $\beta$ [9].

Recently, interleukin family member IL-24 has been attracted researchers attentions by the virtue of the effect on human melanoma cell growth and differentiation [10]. Preclinical studies have shown that IL-24 induces apoptosis in various cancer cells but has no significant cytotoxicity to normal cells [11, 12]. Traditional delivery of IL-24 by liposome or replication-defective adenovirus cannot target tumor cells, which limits its value on cancer gene therapy. Therefore, in this study, we used vaccinia virus strain Guang9 (VG9) as a delivery vector to express IL-24 gene. The antitumor effects and therapeutic potential of VG9-IL-24 for breast cancer were evaluated both in vitro and in vivo.

\section{Materials and Methods}

\section{Cell lines}

The human breast cancer cell lines MDA-MB-231 (TNBC), MDA-MB-453 (TNBC), MDA-MB-468 (TNBC), MCF-7 (ER+, PR+), SK-BR-3 (HER2+), the human normal breast epithelial cell line MCF-10A, and the human embryonic kidney cell line HEK-293 were purchased from Shanghai Cell Collection (Shanghai, China). African green monkey kidney epithelial cell lines Vero and BSC-40 were purchased from the American Type Culture Collection (ATCC; Manassas, VA, USA). All cells were cultured under the conditions suggested by ATCC.

\section{Construction, identification and titration of VG9-IL-24}

The recombinant vaccinia virus carrying IL-24 gene was constructed via homologous recombination between shuttle plasmid ( $\mathrm{pCB}$, gifted from Professor Liu) and vaccinia virus strain VG9 (obtained from National Institutes for Food and Drug Control, Beijing, China), which was derived from Chinese vaccine strain Tian Tan (VTT) and has been demonstrated be more attenuated [13]. The IL-24 gene (full-length cDNA was purchased from Sino Biological Inc. , Beijing, China) was inserted into viral thymidine kinase (TK) locus and was under the control of the vaccinia synthetic early/late promoter
[14]. Recombinants were selected in Vero cells via xanthine-guanine phosphoribosyltransferase (XGPRT) selection. Viral genomes were extracted by Generay kit (Shanghai Generay Biotech Co., Ltd, Shanghai, China). The presence of the inserted IL-24 gene was verified by polymerase chain reaction (PCR) using IL-24 primers (P1: 5'-AGATCTATGAGTTGG GGAC-3'; P2: 5'- GAATTCTCAGAGATGGTAG-3'). A pure recombinant was also verified by PCR using primers external to the site of recombination (P1: 5'ATGAACGGCGGACATATTCA-3'; P2: 5'-TTATGA GTCGATGTAACACTTTC-3'). The results were visualized by ethidium bromide agarose gel electrophoresis. Recombinant vaccinia virus stock were amplified in Vero cells and purified over a sucrose gradient centrifugation. Plaque-forming unit (PFU) virus titers $(\mathrm{PFU} / \mathrm{ml})$ were determined by plaque assay on BSC-40 cells after three cycles of freezing and thawing. The TK-deleted recombinant vaccinia virus expressing enhanced green fluorescent protein (EGFP) gene was generated as control and the construction was described previously [15].

\section{IL-24 expression}

The human breast cancer cell lines and normal breast cells grown in 12-well plates were infected with 0.1 multiplicity of infection (MOI) of VG9-IL-24 for 24 h. Supernatants and lysates were collected and IL-24 levels were determined by enzyme-linked immunosorbent assay (ELISA; R\&D systems Inc., Minneapolis, MN, USA) according to the manufacturer's manual.

\section{Cytotoxicity assay}

The human breast cancer cell lines MDA-MB-231, MDA-MB-453, MDA-MB-468, MCF-7, SK-BR-3, and the human normal breast cell line MCF-10A were seeded in 96-well plates at the density of $1 \times 10^{4} /$ well. After infection with different concentrations of virus for $72 \mathrm{~h}$, cell viability was analyzed by the MTT cytotoxicity assay.

\section{Detection of apoptotic cells}

Hoechst 33258 staining assay was carried out to observe morphological characteristics of apoptotic cells. MDA-MB-231 cells and MCF-10A cells were seeded in 12-well plates. After infection with VG9-IL-24, VG9-EGFP or PBS for $48 \mathrm{~h}$, cells were incubated with Hoechst 33258 (Beyotime Biotechnology, China) for $30 \mathrm{~min}$. The apoptotic morphological changes of cells are observed under Olympus IX51 fluorescence microscope immediately.

The ratios of apoptotic cells were determined by flow cytometric analysis using an Annexin $\mathrm{V} /$ propidium iodide (PI) apoptosis detection kit (Roche Applied Science, Germany). MDA-MB-231 
cells were harvested after infection with VG9-IL-24, VG9-EGFP or PBS for $48 \mathrm{~h}$. Aliquots of cells were resuspended in $1 \mathrm{ml}$ binding buffer mixed with $20 \mu \mathrm{l}$ of fluorescein isothiocyanate (FITC)-labeled Annexin $\mathrm{V}$ and $20 \mu \mathrm{l}$ of PI, and put in the dark at room temperature for $10 \mathrm{~min}$. Flow cytometry (BD, FACSCalibur, USA) was performed immediately after staining.

\section{Cell cycle analysis}

MDA-MB-231 cells seeded in 6-well plates were infected with VG9-IL-24, VG9-EGFP or PBS for $48 \mathrm{~h}$, then were harvested and fixed in $70 \%$ cold ethanol overnight at $-20{ }^{\circ} \mathrm{C}$. Cells were washed with PBS and resuspended in $50 \mu \mathrm{g} / \mathrm{ml}$ of PI solution. After incubation for $30 \mathrm{~min}$ in the dark at $37^{\circ} \mathrm{C}$, the treated cells were analyzed by flow cytometry (BD, FACSCalibur, USA). The percentages of G0/G1, S, and G2/M stage cells were quantified using Flow Jo Software (Tristar, CA, USA).

\section{Western blot analysis}

Cells infected with virus or mock-infected cells were harvested and lysed with RIPA lysis buffer containing protease and phosphatase inhibitors (Halt Protease Inhibitor Cocktail, Thermo Fisher Scientific Inc.). Equal amounts of protein from each sample were subjected to electrophoresis on SDSpolyacrylamide gel and transferred to polyvinylide difluoride membrane (Thermo Scientific ${ }^{\mathrm{TM}}$, USA). Rabbit monoclonal antibody for PI3K (Cell Signaling Technology, Danvers, MA, USA), $\beta$-catenin (Beyotime Institute of Biotechnology, China), phospho-Akt $\left(S^{473}\right)$, Akt (Cell Signaling Technology, Danvers, MA, USA), phospho-GSK-3 $\beta$ (Ser $\left.{ }^{9}\right), \quad$ GSK-3 $\beta$ (Beyotime Institute of Biotechnology, China), Bxl-xL (Beyotime Institute of Biotechnology, China) and mouse monoclonal antibodies for $\beta$-actin (Santa Cruz Biotechnology, Inc., Santa Cruz, CA, USA) were used as primary antibodies. Immunoreactive bands were visualized with chemiluminescence using ECL western blot detection reagents (Santa Cruz Biotechnology, Inc., Santa Cruz, CA, USA).

\section{Animal experiments}

The animal experiment was approved by the Institutional Animal Care and Use Committees (IACUC) of Jiangsu Institute of Nuclear Medicine (JSINM2010007). Female nude BALB/c mice (5-6 weeks old) were purchased from Shanghai Laboratory Animals Center (SLAC; Shanghai, China).

MDA-MB-231 tumor model was established by subcutaneously injecting $5 \times 10^{6}$ cells into the left mammary fat pads of nude mice. When tumors reached the size of $3-5 \mathrm{~mm}$ in diameter, mice were randomly divided into three groups ( $n=6$ per group) and were intratumorally injected with PBS (control group), $10^{7}$ PFU of VG9-IL-24 (VG9-IL-24 group) and $10^{7}$ PFU of VG9-EGFP (VG9-EGFP group). Tumor growth was followed every other day and the tumor volume was calculated as [(width) ${ }^{2} \times$ length] $\times$ $0.52[16]$. Mice were euthanized when tumors reached their maximal permitted size according to the animal regulations, and Kaplan-Meier survival curves were plotted.

\section{HE and immunohistochemical staining}

Tumors in each group were harvested and fixed in $10 \%$ neutral formalin. After conventional paraffin embedding, hematoxylin and eosin (HE) staining and immunohistochemical staining were performed according to standard protocols. HE staining was used to observe morphological changes in tumor tissue cells, such as karyokinetic, which reflect the proliferation activity of tumor cells.

For immunohistochemical analysis, sections were deparaffinized at $60^{\circ} \mathrm{C}$ for $1 \mathrm{~h}$, followed by rehydration, and antigen retrieval using citrate buffer and heating. After treatment with $3 \%$ hydrogen peroxide $\left(\mathrm{H}_{2} \mathrm{O}_{2}\right)$ to block endogenous peroxidase activity and blocking with PBS containing 1\% bovine serum albumin (BSA), sections were washed three times in PBS and incubated for $1 \mathrm{~h}$ at room temperature with Ki-67 (1:100, Novus Biologicals, USA ) or monoclonal anti-IL-24 antibody (1:50, Abcam, Cambridge, UK). Slices were then washed in PBS, incubated with the corresponding secondary antibodies for $30 \mathrm{~min}$ and detected with diaminobenzidine tetrahydrochloride (DAB) solution. After counterstaining with hematoxylin, slices were dehydrated through a sequence of increasing concentrations of alcohol and cleared in xylene. Images were taken with the microscope (magnification, $400 \times$ ).

\section{Statistical analysis}

Statistical analysis was performed by SPSS 19.0 software (SPSS Statistics, Inc., Chicago, IL, USA). Data are presented as mean \pm standard deviation (SD). One-way ANOVA analyses were employed to compare multiple groups, followed by Tukey's test for two groups. Survival analysis was performed using the method of Kaplan-Meier, and differences between curves were assessed using the log-rank test. The $P$ value of less than 0.05 was considered to be statistically significant. 

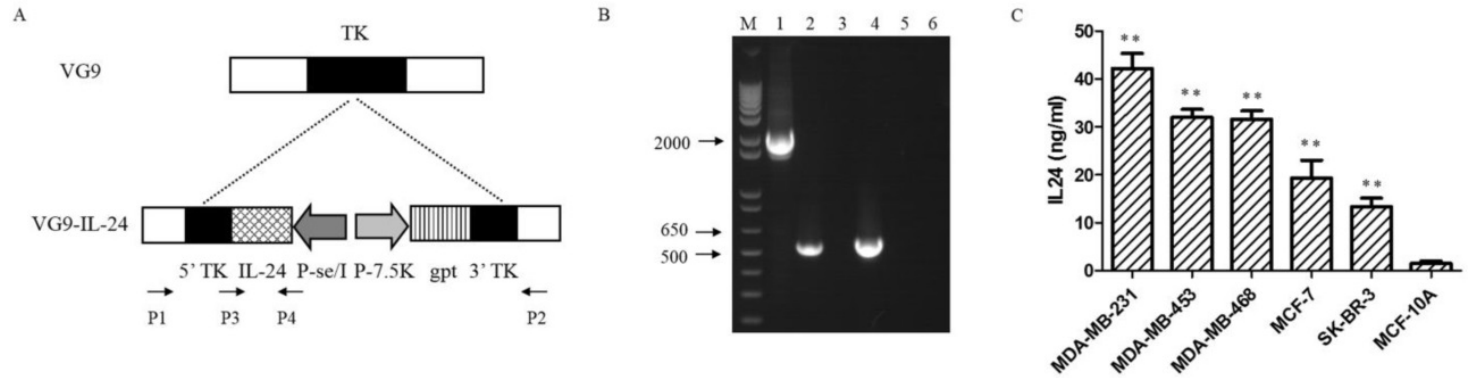

Figure 1. Characterization of VG9-IL-24. (A) Schematic illustration of VG9-IL-24 construction. The IL-24 gene was inserted into TK locus of VG9 strain via homologous recombination. (B) Identification of VG9-IL-24 by PCR analysis. M, IKb Plus DNA Ladder (Invitrogen); Lane 1 (VG9-IL-24), P1/P2 primers amplify a 2020-bp fragment across the region of recombination, which confirm absence of TK. The TK positive fragment (534-bp) amplified of VG9 is shown in Lane 3 and negative control $\left(\mathrm{H}_{2} \mathrm{O}\right)$ is shown in Lane 5. Lane 2 (VG9-IL-24), P3/P4 primers amplify IL-24 to product a 546-bp band, which is absent in VG9 (Lane 4). Negative control $\left(\mathrm{H}_{2} \mathrm{O}\right)$ is shown in Lane 6. (C) Protein concentrations of IL-24 in breast malignant and normal cells. The concentrations of IL-24 protein from all breast cancer cells treated with VG9-IL-24 was higher than that in normal cells (all $P<0.01)$. Each bar represents the mean $\pm S D(n=3)$. $* * P<0.01$.

\section{Results}

\section{Construction and characterization of VG9-IL-24}

VG9-IL-24 was generated by inserting the IL-24 gene into TK locus of vaccinia strain VG9 by homologous recombination (Figure 1A). PCR analysis using specific primers designed were performed to detect the wild-type virus and confirmed the insertion of IL-24 gene (Figure 1B). To further confirm the exogenous IL-24 expression, protein in supernatants and lysates from breast cancer cells and normal cells infected with VG9-IL-24 were harvested and quantified by ELISA. As expected, the concentrations of IL-24 protein from all breast cancer cells treated with VG9-IL-24 was remarkably increased compared with that from normal cells (all $P<0.01$; Figure 1C). Endogenous IL-24 expression was not detected in cells treated with VG9-EGFP or PBS control groups (data not shown).

\section{Oncolytic activity of VG9-IL-24 in vitro}

Viral proliferation was assessed on breast malignant and normal cells. After infection of VG9-IL-24 at MOI of 0.1, samples were collected at various times. We found that VG9-IL-24 replicated rapidly in MDA-MB-231 cells and the titer was markedly higher than that in MCF-10A cells at indicated times (all $P<0.05$; Figure 2A). To further investigate the selective killing of VG9-IL-24 on tumor cells, various breast cancer cell lines and normal cells were infected with increasing doses of virus and cell viability was assessed by MTT. As shown in Figure $2 \mathrm{~B}$, the cytotoxic effect was obvious on breast cancer cell lines with diverse sensitivity to different cell lines, but there was no significant cytotoxicity on normal cells. At MOI of 1, more than $50 \%$ of all breast cancer cells were killed. At MOI of 10 , less than $20 \%$ of MCF-7 and MDA-MB-231 cells survived and about $20 \%-40 \%$ in other breast cancer cell lines. However,
VG9-IL-24 had little cytotoxic effect on normal cells.

These results all indicate that VG9-IL-24 can selectively replicate in breast cancer cell lines and has oncolytic potency on tumor cells without significant cytotoxicity to normal cells.

\section{VG9-IL-24-mediated apoptosis in breast cancer cells}

VG9-IL-24 induced apoptosis in breast cancer cells was first assessed by Hoechst staining to observe apoptotic morphological changes. As shown in Figure $3 \mathrm{~A}$, nuclear fragmentation and chromatin clumping were evidently observed in MDA-MB-231 cells infected with VG9-IL-24 for $48 \mathrm{~h}$, but no obvious apoptotic changes showed in normal cells. No significant changes were observed in any of the cells treated with PBS.

Annexin-V and PI staining assays with flow cytometry were then performed to quantify the apoptosis induction in tumor cells (Figure 3B). After infection with VG9-IL-24, VG9-EGFP and PBS, the apoptosis ratios of MDA-MB-231 cells were $(95.62 \pm 0.86) \%,(54.89 \pm 1.52) \%$ and $(6.29 \pm 0.88) \%$, respectively. Compared with VG9-EGFP and PBS group, the number of apoptotic cells was obviously increased in VG9-IL-24 group $(P<0.01$ compared with PBS group; $P<0.05$ compared with VG9-EGFP group).

Previous studies have demonstrated that IL-24 is able to induce $\mathrm{G} 2 / \mathrm{M}$ cell-cycle arrest in various cancer cell lines [12, 17-19]. To determine whether VG9-IL-24 induces G2/M accumulation in MDA-MB-231 cells, cell-cycle phases were analyzed by flow cytometry. Results showed that VG9-IL-24 induced higher $\mathrm{G} 2 / \mathrm{M}$ proportion of the cell cycle in MDA-MB-231 cells compared with PBS $(P<0.01)$ and VG9-EGFP $(P<0.05$; Figure $3 C)$.

Together, these results indicated that VG9-IL-24 notably inhibited growth, stimulated the apoptosis of breast cancer cells, and arrested breast cancer cells in the G2/M phase. 


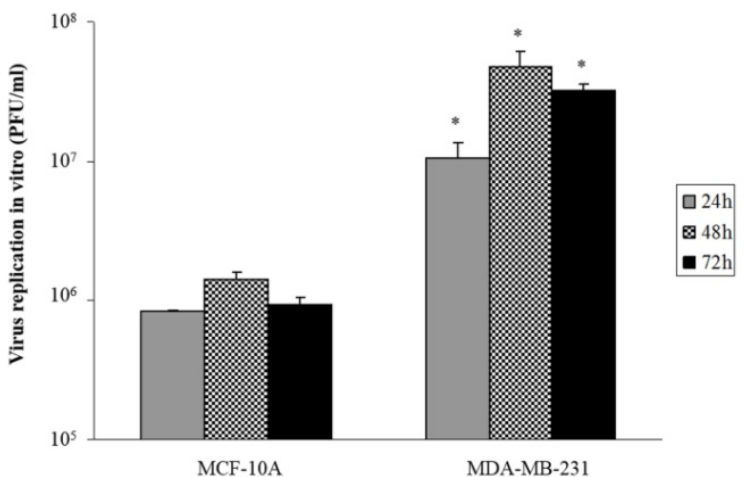

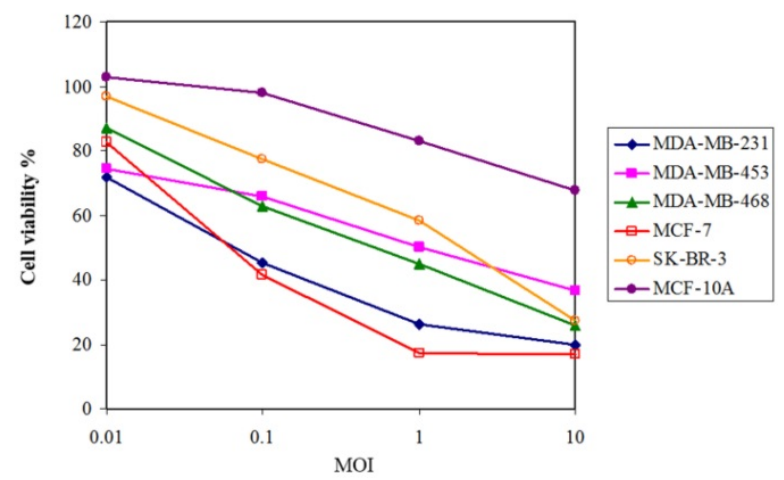

Figure 2. Oncolytic activity of VG9-IL-24. (A) Selective replication of VG9-IL-24 in breast cancer cells. MDA-MB-231 cells and MCF-10A cells in 12-well plates were infected with VG9-IL-24 at $0.1 \mathrm{MOI}$ and samples were collected at indicated times. Virus titers in MDA-MB-231 cells were higher than those in MCF-10A cells at indicated times (all $P<0.05)$. Each bar represents the mean $\pm S D(n=3)$. $* P<0.05$. (B) Breast cancer cell lines and normal cells were infected with VG9-IL-24 at various MOls. After infection for $72 \mathrm{~h}$, cell viability was measured by MTT assay. The cytotoxic effect was obvious on breast cancer cell lines, while little cytotoxicity on normal cells.

A

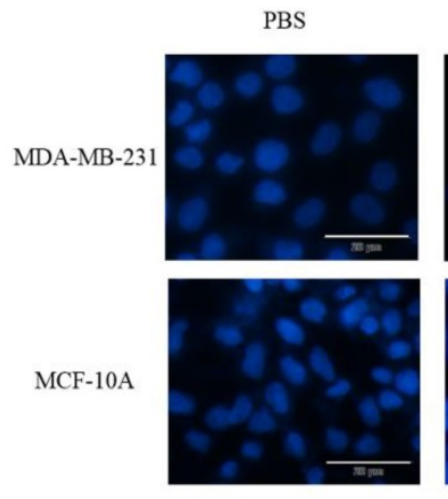

VG9-IL-24
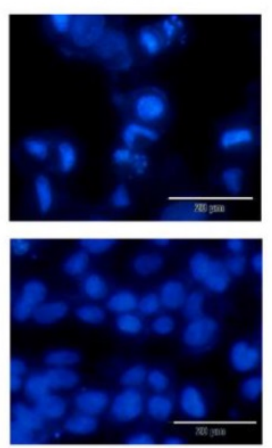

VG9-EGFP
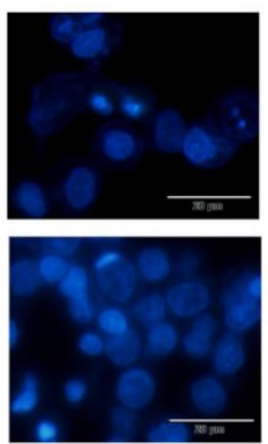

B
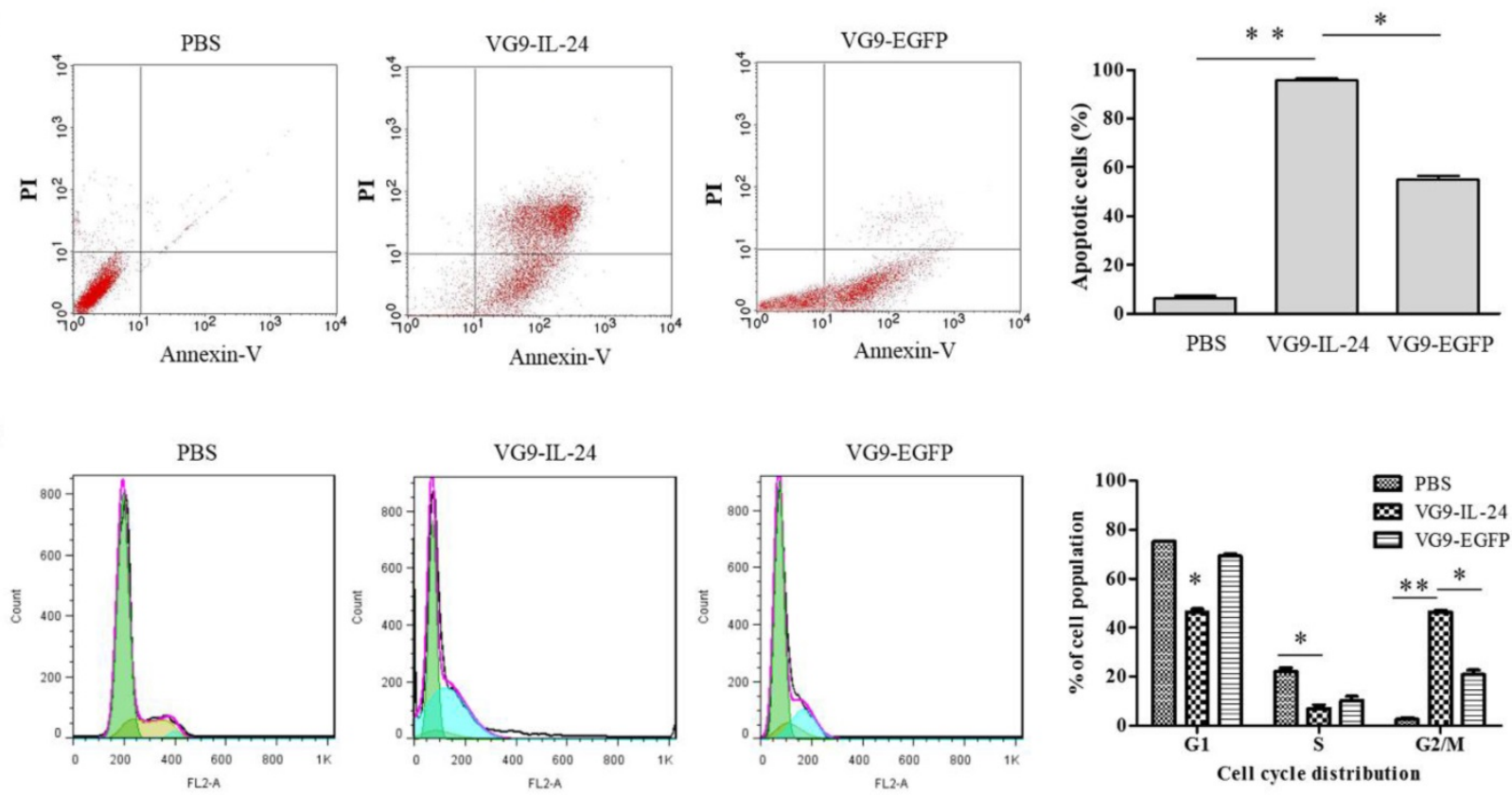

Figure 3. VG9-IL-24 induced apoptosis of breast cancer cells. (A) Cell apoptotic staining by Hoechst 33258. MDA-MB-231 cells and MCF-10A cells treated with PBS, VG9-IL-24 or VG9-EGFP were incubated with Hoechst 33258 for 30 min, and nuclear fragmentation and chromatin clumping were observed in virus-treated groups but not in normal cells. Bar: $20 \mu \mathrm{m}$. (B) The percentage of apoptotic cells was determined by flow cytometry. MDA-MB-231 cells treated with PBS, VG9-IL-24 or VG9-EGFP were harvested after $48 \mathrm{~h}$ and stained with FITC-labeled Annexin $\mathrm{V}$ and PI and immediately analyzed by flow cytometry. The apoptosis ratio of VG9-IL-24 group was significantly higher than that of VG9-EGFP or PBS group $(P<0.01$ compared with PBS group; $P<0.05$ compared with VG9-EGFP group). (C) Cell-cycle analysis by flow cytometry. MDA-MB-231 cells treated with PBS, VG9-IL-24 or VG9-EGFP were harvested after $48 \mathrm{~h}$ and stained with PI. Cell cycle distribution was analyzed by flow cytometry and the percentage of cell-cycle phases was analyzed. Higher G2/M proportion of the cell cycle was observed in VG9-IL-24 group compared with PBS or VG9-EGFP group ( $P<0.01$ compared with PBS group; $P<0.05$ compared with VG9-EGFP group). Each bar represents the mean \pm SD of three independent experiments. $* P<0.05 ; * * P<0.01$. 
A

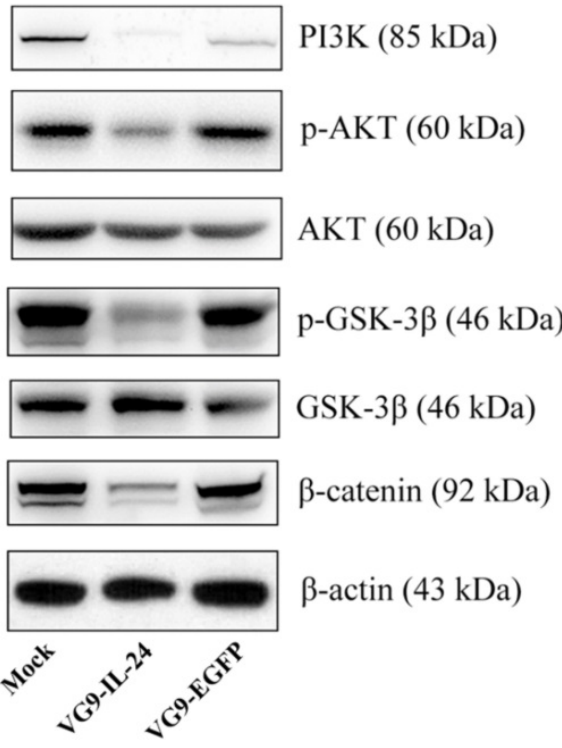

B

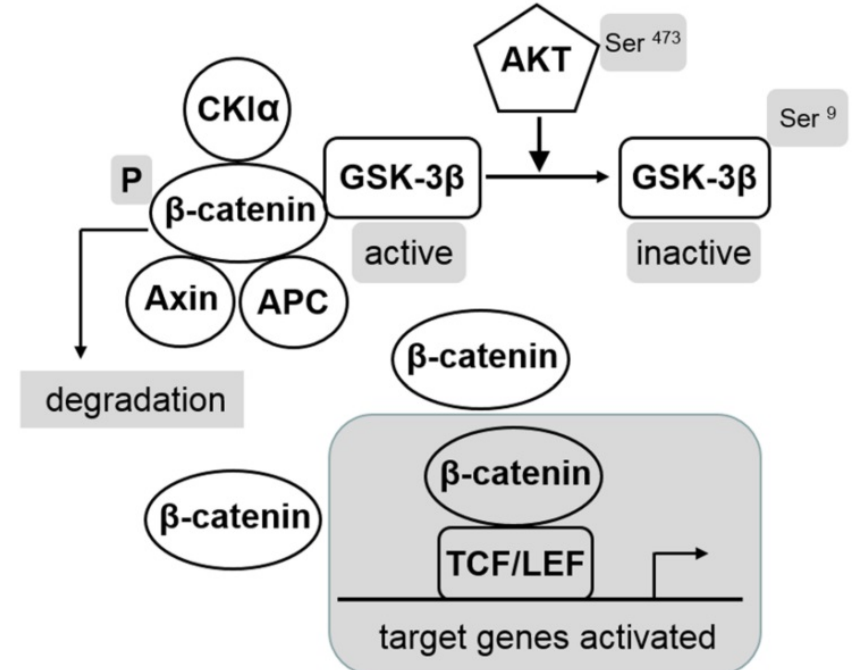

Figure 4. VG9-IL-24 induced apoptosis in breast cancer cells via PI3K/ 3 -catenin signaling pathway. (A) MDA-MB-231 cells treated with PBS, VG9-IL-24, VG9-EGFP for $48 \mathrm{~h}$ were harvested, lysed and prepared to be available for western blot analysis. Reduced expressions of PI3K, phosphorylation of Akt and GSK-3 $\beta$, and $\beta$-catenin were observed in VG9-IL-24-treated cells. $\beta$-actin was used as a loading control. (B) Schematic illustration of VG9-IL-24 regulation of PI3K/ $\beta$-catenin signaling pathway.

\section{VG9-IL-24 regulates $P I 3 K / \beta$-catenin signaling pathway in breast cancer cells}

IL-24 negatively regulating the $\mathrm{PI} 3 \mathrm{~K} / \beta$-catenin pathway was observed in breast cancer cells previously [20]. To determine whether the apoptotic effect of VG9-IL-24 on breast cancer cells was related to $\mathrm{PI} 3 \mathrm{~K} / \beta$-catenin signaling pathway, we investigated the activity of VG9-IL-24 on the representative signal proteins of this pathway. As shown in Figure 4A, MDA-MB-231 cells infected with VG9-IL-24 showed reduced levels of PI3K, which resulted in down-regulation of phosphorylation of protein kinase B (Akt, Ser $\left.{ }^{473}\right)$ and subsequent decrease of phosphorylation of glycogen synthase kinase $3 \beta$ (GSK-3 $\beta$, Ser ${ }^{9}$ ) and increase of GSK-3 $\beta$. As the negative regulator of $\beta$-catenin, activated GSK-3 $\beta$ led to decreased level of $\beta$-catenin protein due to ubiquitin-proteasome degradation by phosphorylation. Western blotting results suggested that VG9-IL-24 induced apoptosis in breast cancer cells might via $\mathrm{PI} 3 \mathrm{~K} \rightarrow \mathrm{AKT} \rightarrow \mathrm{GSK}-3 \beta \rightarrow \beta$-catenin pathway (Figure 4B).

\section{Antitumor effect of VG9-IL-24 in vivo}

To further evaluate the antitumor effect of VG9-IL-24 in vivo, MDA-MB-231 xenograft mouse model was established. When tumors reached 3 to 5 $\mathrm{mm}$ in diameter, VG9-IL-24, VG9-EGFP or PBS (control) was intratumorally injected. Results showed that tumors grew progressively in control group, while virus-treated mice exhibited significant suppression of tumor development. By 40 days post-treatment, an increased tumor growth was observed in the VG9-EGFP group, but tumor growth was delayed in the VG9-IL-24 group (Figure 5A). All control mice died within 40 days, however, VG9-IL-24-treated mice survived extended up to 70 days with survival rate of $60 \%$ (Figure 5B).

$\mathrm{HE}$ and immunohistochemical staining provided further evidence of the antitumor activity of VG9-IL-24. As shown in Figure 6, apparent karyopyknosis, cytoplasm concentration and dissolution of the nucleus were observed in VG9-IL-24 group by HE staining. Immunohistochemical analysis for Ki-67, a tumor cell proliferation marker that positively correlates with prognosis in various malignant tumors, showed that VG9-IL-24 significantly inhibited the proliferation of tumor cells. Immunohistochemical staining also confirmed that IL-24 was stably expressed in tumor tissue from VG9-IL-24-treated group. These results indicated that VG9-IL-24 was able to efficiently generate IL-24 protein, resulting in inhibition of tumor cells proliferation. 


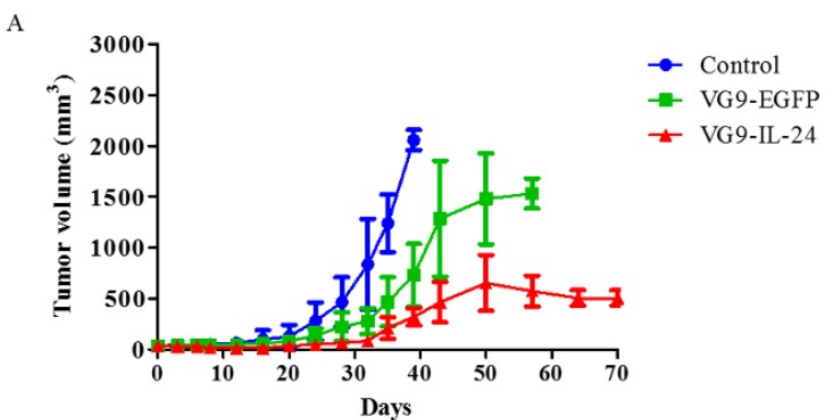

B

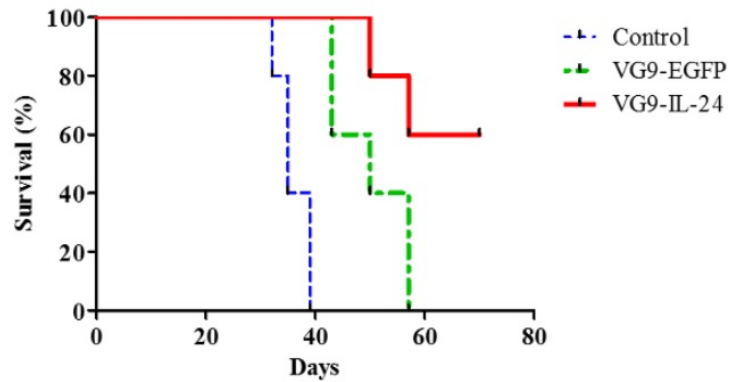

Figure 5. Antitumor effect of VG9-IL-24 in MDA-MB-231 xenograft mouse model. (A) Mean tumor volume in mice treated with PBS (Control), VG9-IL-24, or VG9-EGFP. Tumors developed rapidly in Control and VG9-EGFP groups, while tumor growth was significantly slow in VG9-IL-24 group. $\mathrm{n}=6$ in each group. (B) Kaplan-Meier survival curves for tumor-bearing mice treated with PBS, VG9-IL-24, or VG9-EGFP. Higher survival rate was observed in VG9-IL-24 group compared with Control or VG9-EGFP group. $n=6$ in each group.

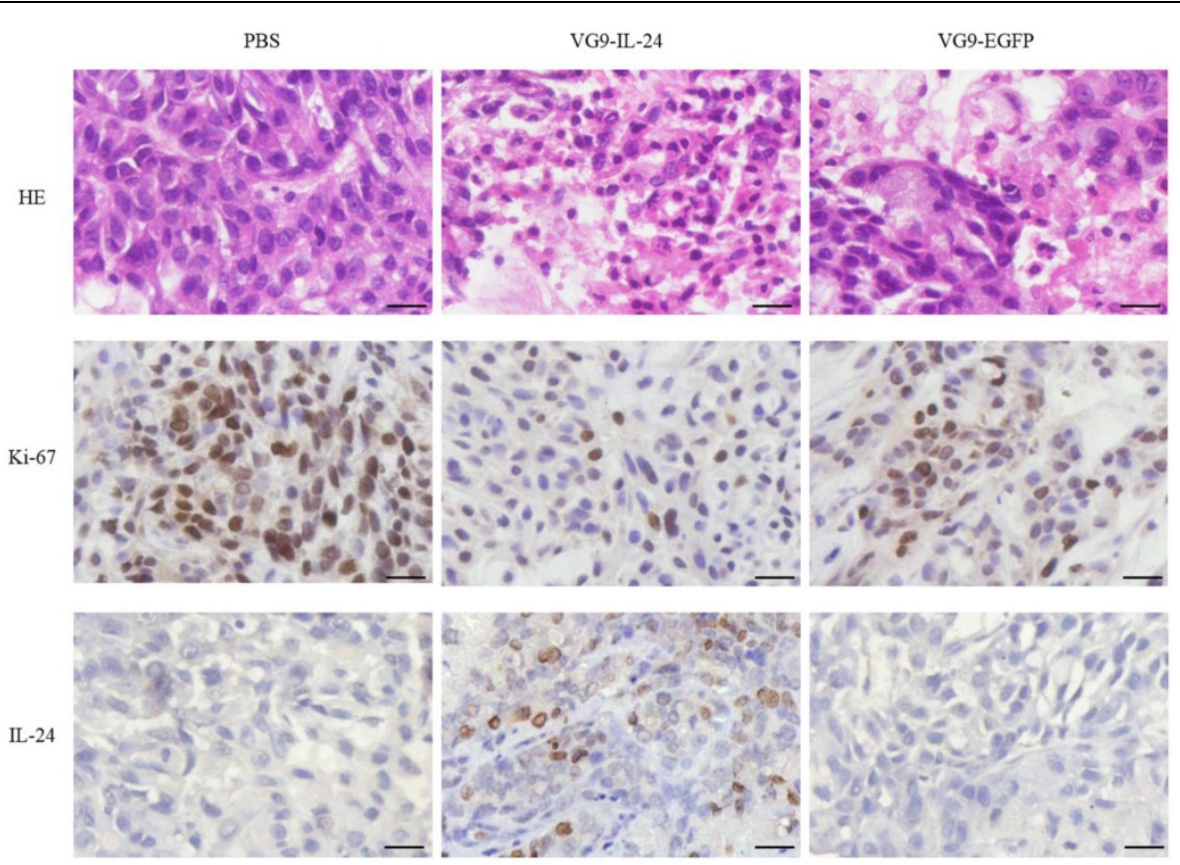

Figure 6. HE and immunohistochemical staining of the tumor tissue. Tumors from the mice treated with PBS (Control), VG9-IL-24, or VG9-EGFP were harvested, formalin fixed and paraffin embedded. Sections were subjected to HE staining and immunohistochemistry for Ki-67 and IL-24. Pyknosis events were obviously appeared in VG9-IL-24 group and decreased positive Ki-67 were also observed in VG9-IL-24 group. Expression of IL-24 was observed in tumors treated with VG9-IL-24. Original magnification, $\times 400$. Bar: $20 \mu \mathrm{m}$.

\section{Discussion}

Targeted oncolytic vaccinia virus has emerged as a novel therapeutic strategy for cancer in recent years. Due to direct tumor cells lysis by the virtue of selective replication in tumor cells, oncolytic vaccinia virus are potential to treat a wide variety of cancers, including breast cancer. In present study, we constructed the oncolytic vaccinia virus harbored with IL-24 (VG9-IL-24). High and stable expression of IL-24 was available with the replication of vaccinia virus confirmed by ELISA and immunohistochemical staining. The antitumor effects of this recombinant vaccinia virus were significant both in vitro and in vivo.

Vaccinia virus has been used as a live vaccine in the smallpox eradication with a long history, which making it safe and easy to use. Recently, it as has been used as vaccine against cancer due to multiple favorable attributes. Vaccinia virus replicates and lyses cells rapidly compared with other virus species. It entries into target cells through several membrane fusion pathways within $2 \mathrm{~h}$ [21], and spends its entire lifecycle in the cytoplasm, allowing for rapid, efficient replication without negative effects from host cell defenses. The first viral particles produced are secreted from cells within $8 \mathrm{~h}$ and infected cells are destroyed $48 \mathrm{~h}$ to $72 \mathrm{~h}$ after infection [22]. With infected cells dying, the viral production decreased. Similarly in our study, VG9-IL-24 replicated rapidly, reaching a maximum within $48 \mathrm{~h}$ and the value slightly changed by $72 \mathrm{~h}$.

Many studies and clinic trails have been examined the applicability of several vaccine strains such as Wyeth [23, 24], Copenhagen [25] and Lister 
[26]. However, the researches on Chinese vaccine strain as oncolytic agent were few. The vaccinia virus strain VTT was the most widely used vaccine in China and the biological characteristics have already been studied systematically [27]. VTT is less virulent than vaccinia virus strain WR [27], which has been widely used in laboratories and extensively tested in clinical trials. VG9 was derived from VTT by using consecutive plaque-cloning selection and has been demonstrated smaller necrosis area and pock diameter production, less red swelling and lower incidences of fever and hyperpyrexia [13]. Although VG9 still had neurotoxicity to a certain extent, the virulence was found to be lower than VTT in various animal models. Therefore, VG9 is supposed to be a promising vaccinia virus vector.

Replication oncolytic vaccinia virus exerts attractive antitumor effects not only through the infection and lysis of cancer cells, but also through expression of various therapeutic transgenes. JX-594, a Wyeth strain armed with GM-CSF, has demonstrated eradication of lung metastases from liver tumors in rabbits [5] and potential antitumor effects both at the injection site and in distant non-injected tumors in advanced hepatocellular carcinoma patients [28, 29]. WR strain carrying IL-12 also showed efficient infection in a variety of tumor cell lines and significant reduction in tumor growth [6]. The therapeutic gene engineered in this study was IL-24, a novel cancer growth-suppressing and apoptosis-inducing gene, which has been demonstrated efficient and safe in preclinical studies based on adenovirus [30-33]. Studies have shown that IL-24 can suppresses cell growth and induce apoptosis in a variety of tumor types [17, 30, 34-37]. Our data showed that vaccinia virus-mediated IL-24 exerts strong killing effects on various breast cancer cell lines; of note, it also has the cytotoxic effect on TNBC cells, such as MDA-MB-231, MDA-MB-453 and MDA-MB-468 cells. In contrast, overexpression of IL-24 in normal cells has no significant cytotoxicity [12]; similar results were observed in our study that normal breast epithelial cell line MCF-10A exerts no injurious effects.

According to that IL-24 is a prospective tumor suppressor cytokine for multiple cancers, it is worth to explore the mechanism by which IL-24 induces apoptosis selectively in cancer cells. However, the mechanism is complicated and signaling pathways involved are different and seem to be dependent on tumor types [38]. Previous studies have revealed that multiple signaling pathways and intracellular molecules play a role in IL-24-mediated apoptosis including activation of the p38 MAPK pathway [39], inhibition of the Wnt/PI3K pathways [20], activation of the Fas-FasL signaling pathway [40], activation of caspase cascade [41], activation of RNA-dependent protein kinase R (PKR) [42] and c-Jun-NH2-kinase [43], downregulation of anti-apoptotic proteins [44], as well as upregulation of pro-apoptotic proteins [30]. In this study, we investigated that the apoptotic effect of VG9-IL-24 on breast cancer cells was related to $\mathrm{PI} 3 \mathrm{~K} / \beta$-catenin signaling pathways. Results showed that VG9-IL-24 inhibited expression of oncogenic protein PI3K, thus decreased the phosphorylation of Akt $\left(\right.$ Ser $\left.^{473}\right)$, which is known as a major downstream mediator of the PI3K pathway. As the downstream target of Akt, phosphorylation of GSK-3 $\beta$ (Ser ${ }^{9}$ ) downregulated due to Akt kinase activity inhibition, thereby maintaining GSK-3 $\beta$ in its active form which activated the destruction complex (constituted with $\beta$-catenin, adenomatous polyposis coli, casein kinase $1 \alpha / a x i n$, and GSK-3 $\beta$ ) to phosphorylate $\beta$-catenin $\left(\mathrm{Ser}^{33} / \mathrm{Ser}^{37} / \mathrm{Thr}^{41}\right.$ ), thus marking it for proteasome-mediated degradation. As a consequence, cytosolic pools of $\beta$-catenin reduced and no more translocated into the nucleus to bind the transcription factor T-cell factor/lymphoid enhancer factor (TCF/LEF), leading to inhibiting expression of TCF/LEF-responsive genes which function in cell cycle progression and loss of cell differentiation.

The apoptotic effects of VG9-IL-24 were also investigated by Hoechst staining, flow cytometric analysis, and cell cycle analysis. After VG9-IL-24 treatment, nuclear fragmentation and chromatin clumping in breast cancer cells were observed, apoptotic cell ratio was increased, and accumulation of cells in the G2/M phase of the cell cycle was determined. To further evaluate the antitumor effect of VG9-IL-24 in vivo, we established an orthotopic breast cancer model by injecting MDA-MB-231 cells into the mammary fat pads of nude mice. Results showed that tumor growth in VG9-IL-24-treated mice was notably slower compared with VG9-EGFP or control group and the survival rate of VG9-IL-24 group was $60 \%$, which was similar with the other recombinant vaccinia virus carrying IL-12 in previous study [6]. HE and immunohistochemical analysis results indicated that tumor cells treated with VG9-IL-24 underwent apoptosis, while apoptosis was not detected in control group or found in only a small amount of tumor cells treated with VG9-EGFP, which was consistent with the results in vitro study.

As a novel tumor suppressor cytokine, IL-24 has multifaceted antitumor effects. In addition to selectively suppressing growth and inducing apoptosis of tumor cells, other antitumor features including inhibition of tumor cell invasion and metastasis, anti-angiogenic activity, immune modulatory activity, and "bystander" antitumor activity were also observed [45-48]. In present study, 
we only investigated selective apoptosis induction of VG9-IL-24 on breast cancer cells in vitro and xenograft nude mice in vivo. To further confirm whether VG9-IL-24 could induce antitumor immunity and "bystander" antitumor effect, immune competent mice models should be established in future study.

Collectively, we constructed a recombinant vaccinia virus VG9-IL-24, which was armed with a potent tumor-suppressing gene IL-24, and demonstrated it exerted significant antitumor effects on breast cancer both in vitro and in vivo. Beyond direct cell lysis, VG9-IL-24 also induced apoptosis in breast cancer cells without harming normal cells. Our findings suggest that VG9-IL-24 holds a significant promise as an innovative therapy for breast cancer in clinic trails.

\section{Abbreviations}

IL-24: Interleukin-24; VG9: Vaccinia Strain Guang9; VTT: Vaccine Strain Tian Tan; TK: Thymidine Kinase; XGPRT: xanthine-guanine phosphoribosyltransferase; PFU: Plaque-forming Unit; EGFP: enhanced green fluorescent protein; MOI: Multiplicity of Infection; ELISA: Enzyme-linked Immunosorbent Assay; PI: Propidium Iodide; PI3K: Phosphoinositide 3-kinase; AKT: protein kinase B; GSK-3 $\beta$ : glycogen synthase kinase $3 \beta$; HE: hematoxylin and eosin.

\section{Acknowledgements}

We are grateful to Professor Xinyuan Liu for providing the vaccinia shuttle plasmid ( $\mathrm{pCB}$ ), and the National Institutes for Food and Drug Control (NIFDC) for providing the vaccinia virus of Tian Tan strain VG9. This work was supported by the National Natural Science Foundation of China (81703061), Innovation Capacity Development Plan of Jiangsu Province (BM2018023) and Jiangsu Provincial Key Medical Discipline (ZDXKA2016017).

\section{Ethical approval}

The animal experiment was approved by the Institutional Animal Care and Use Committees (IACUC) of Jiangsu Institute of Nuclear Medicine (JSINM2010007). All procedures were performed in accordance with the Laboratory animal-Guideline of welfare ethical review of Chinese IACUC.

\section{Availability of data and material}

The datasets during and/or analyzed during the current study are available from the corresponding author on reasonable request.

\section{Authors' contributions}

LD contributed to study conception, recombinant vaccinia virus construction, cytotoxic assay, funding acquisition, and original draft writing. JF generated tumor model and performed animal study. YD carried out cell cycle analysis, western blot analysis and in vivo experiments. $X Y$ performed viral replication and immunohistochemical staining. $\mathrm{BH}$ contributed to study conception, review and editing. $\mathrm{ZH}$ performed statistical analysis and editing.

\section{Competing Interests}

The authors have declared that no competing interest exists.

\section{References}

1. Siegel RL, Miller KD, Jemal A. Cancer statistics, 2016. CA Cancer J Clin. 2016; 66: 7-30.

2. Kirn DH, Thorne SH. Targeted and armed oncolytic poxviruses: a novel multi-mechanistic therapeutic class for cancer. Nat Rev Cancer. 2009; 9: 64-71.

3. Kim M. Replicating poxviruses for human cancer therapy. J Microbiol. 2015; 53: 209-18.

4. Guse K, Cerullo V, Hemminki A. Oncolytic vaccinia virus for the treatment of cancer. Expert Opin Biol Ther. 2011; 11: 595-608.

5. Kim JH, Oh JY, Park BH, Lee DE, Kim JS, Park HE, et al. Systemic armed oncolytic and immunologic therapy for cancer with JX-594, a targeted poxvirus expressing GM-CSF. Mol Ther. 2006: 14:361-70.

6. Meko JB, Yim JH, Tsung K, Norton JA. High cytokine production and effective antitumor activity of a recombinant vaccinia virus encoding murine interleukin 12. Cancer Res. 1995; 55: 4765-70.

7. Elkins KL, Ennist DL, Winegar RK, Weir JP. In vivo delivery of interleukin-4 by a recombinant vaccinia virus prevents tumor development in mice. Hum Gene Ther. 1994; 5: 809-20.

8. Kurilla MG, Swaminathan S, Welsh RM, Kieff E, Brutkiewicz RR. Effects of virally expressed interleukin-10 on vaccinia virus infection in mice. J Virol. 1993; 67: 7623-8

9. Kirn DH, Wang Y, Le Boeuf F, Bell J, Thorne SH. Targeting of interferon-beta to produce a specific, multi-mechanistic oncolytic vaccinia virus. PLoS Med. 2007; 4: e353.

10. Wang $\mathrm{M}$, Tan $\mathrm{Z}$, Zhang $\mathrm{R}$, Kotenko SV, Liang $\mathrm{P}$. Interleukin 24 (MDA-7/MOB-5) signals through two heterodimeric receptors, IL-22R1/IL-20R2 and IL-20R1/IL-20R2. J Biol Chem. 2002; 277: 7341-7.

11. Sauane M, Gopalkrishnan RV, Sarkar D, Su ZZ, Lebedeva IV, Dent P, et al. MDA-7/IL-24: novel cancer growth suppressing and apoptosis inducing cytokine. Cytokine Growth Factor Rev. 2003; 14: 35-51.

12. Lebedeva IV, Su ZZ, Chang Y, Kitada S, Reed JC, Fisher PB. The cancer growth suppressing gene mda-7 induces apoptosis selectively in human melanoma cells. Oncogene. 2002; 21: 708-18.

13. Zhu R, Liu Q, Huang W, Yu Y, Wang Y. Comparison of the replication characteristics of vaccinia virus strains Guang 9 and Tian Tan in vivo and in vitro. Arch Virol. 2014 1 159: 2587-96.

14. Chakrabarti S, Sisler JR, Moss B. Compact, synthetic, vaccinia virus early/late promoter for protein expression. Biotechniques. 1997; 23: 1094-7.

15. Deng L, Fan J, Ding Y, Zhang J, Zhou B, Zhang Y, et al. Oncolytic efficacy of thymidine kinase-deleted vaccinia virus strain Guang9. Oncotarget. 2017; 8: 40533-43.

16. O'Reilly MS, Boehm T, Shing Y, Fukai N, Vasios G, Lane WS, et al. Endostatin: an endogenous inhibitor of angiogenesis and tumor growth. Cell. 1997; 88: 277-85.

17. Saeki T, Mhashilkar A, Chada S, Branch C, Roth JA, Ramesh R. Tumor-suppressive effects by adenovirus-mediated mda-7 gene transfer in non-small cell lung cancer cell in vitro. Gene Ther. 2000; 7: 2051-7.

18. Saito Y, Miyahara R, Gopalan B, Litvak A, Inoue S, Shanker M, et al. Selective induction of cell cycle arrest and apoptosis in human prostate cancer cells through adenoviral transfer of the melanoma differentiation-associated -7 (mda-7)/interleukin-24 (IL-24) gene. Cancer Gene Ther. 2005; 12: 238-47.

19. Ekmekcioglu S, Ellerhorst J, Mhashilkar AM, Sahin AA, Read CM, Prieto VG, et al. Down-regulated melanoma differentiation associated gene (mda-7) expression in human melanomas. Int J Cancer. 2001; 94: 54-9.

20. Mhashilkar AM, Stewart AL, Sieger K, Yang HY, Khimani AH, Ito I, et al. MDA-7 negatively regulates the beta-catenin and PI3K signaling pathways in breast and lung tumor cells. Mol Ther. 2003; 8: 207-19.

21. Doms RW, Blumenthal R, Moss B. Fusion of intra- and extracellular forms of vaccinia virus with the cell membrane. J Virol. 1990; 64: 4884-92.

22. Zeh HI, Bartlett DL. Development of a replication-selective, oncolytic poxvirus for the treatment of human cancers. Cancer Gene Ther. 2002; 9: 1001-12. 
23. Hwang TH, Moon A, Burke J, Ribas A, Stephenson J, Breitbach CJ, et al. A mechanistic proof-of-concept clinical trial with JX-594, a targeted multi-mechanistic oncolytic poxvirus, in patients with metastatic melanoma. Mol Ther. 2011; 19: 1913-22.

24. Thorne SH, Hwang TH, O'Gorman WE, Bartlett DL, Sei S, Kanji F, et al. Rational strain selection and engineering creates a broad-spectrum, systemically effective oncolytic poxvirus, JX-963. J Clin Invest. 2007; 117: 3350-8.

25. Foloppe J, Kintz J, Futin N, Findeli A, Cordier P, Schlesinger Y, et al. Targeted delivery of a suicide gene to human colorectal tumors by a conditionally replicating vaccinia virus. Gene Ther. 2008; 15: 1361-71.

26. Zhang Q, Yu YA, Wang E, Chen N, Danner RL, Munson PJ, et al. Eradication of solid human breast tumors in nude mice with an intravenously injected light-emitting oncolytic vaccinia virus. Cancer Res. 2007; 67: 10038-46.

27. Fang Q, Yang L, Zhu W, Liu L, Wang H, Yu W, et al. Host range, growth property, and virulence of the smallpox vaccine: vaccinia virus Tian Tan strain. Virology. 2005; 335: 242-51.

28. Park BH, Hwang T, Liu TC, Sze DY, Kim JS, Kwon HC, et al. Use of a targeted oncolytic poxvirus, JX-594, in patients with refractory primary or metastatic liver cancer: a phase I trial. Lancet Oncol. 2008; 9: 533-42.

29. Liu TC, Hwang T, Park BH, Bell J, Kirn DH. The targeted oncolytic poxvirus JX-594 demonstrates antitumoral, antivascular, and anti-HBV activities in patients with hepatocellular carcinoma. Mol Ther. 2008; 16: 1637-42.

30. Zhao L, Gu J, Dong A, Zhang Y, Zhong L, He L, et al. Potent antitumor activity of oncolytic adenovirus expressing mda-7/IL-24 for colorectal cancer. Hum Gene Ther. 2005; 16: 845-58.

31. Qian W, Liu J, Tong Y, Yan S, Yang C, Yang M, et al. Enhanced antitumor activity by a selective conditionally replicating adenovirus combining with MDA-7/interleukin-24 for B-lymphoblastic leukemia via induction of apoptosis. Leukemia. 2008; 22: 361-9.

32. Yang C, Tong Y, Ni W, Liu J, Xu W, Li L, et al. Inhibition of autophagy induced by overexpression of mda-7/interleukin-24 strongly augments the antileukemia activity in vitro and in vivo. Cancer Gene Ther. 2010; 17: 109-19.

33. Zhong S, Yu D, Wang Y, Oiu S, Wu S, Liu XY. An armed oncolytic adenovirus ZD55-IL-24 combined with ADM or DDP demonstrated enhanced antitumor effect in lung cancer. Acta Oncol. 2010; 49: 91-9.

34. Leath CA, 3rd, Kataram M, Bhagavatula P, Gopalkrishnan RV, Dent P, Fisher $\mathrm{PB}$, et al. Infectivity enhanced adenoviral-mediated mda-7/IL-24 gene therapy for ovarian carcinoma. Gynecol Oncol. 2004; 94: 352-62.

35. Zhu W, Wei L, Zhang H, Chen J, Qin X. Oncolytic adenovirus armed with IL-24 inhibits the growth of breast cancer in vitro and in vivo. J Exp Clin Cancer Res. 2012; 31: 51

36. Liu Z, Xu L, Yuan H, Zhang Y, Zhang X, Zhao D. Oncolytic adenovirusmediated mda7/IL24 expression suppresses osteosarcoma growth and enhances sensitivity to doxorubicin. Mol Med Rep. 2015; 12: 6358-64.

37. Zheng SY, Ge JF, Zhao J, Jiang D, Li F. Adenovirus-mediated IL-24 confers radiosensitization to human lung adenocarcinoma in vitro and in vivo. Mol Biol Rep. 2015; 42: 1069-80.

38. Sarkar D, Su ZZ, Lebedeva IV, Sauane M, Gopalkrishnan RV, Dent P, et al. mda-7 (IL-24): signaling and functional roles. Biotechniques. 2002; Suppl: 30-9.

39. Sarkar D, Su ZZ, Lebedeva IV, Sauane M, Gopalkrishnan RV, Valerie K, et al. mda-7 (IL-24) Mediates selective apoptosis in human melanoma cells by inducing the coordinated overexpression of the GADD family of genes by means of p38 MAPK. Proc Natl Acad Sci U S A. 2002; 99: 10054-9.

40. Gopalan B, Litvak A, Sharma S, Mhashilkar AM, Chada S, Ramesh R. Activation of the Fas-FasL signaling pathway by MDA-7/IL-24 kills human ovarian cancer cells. Cancer Res. 2005; 65: 3017-24.

41. Yacoub A, Park MA, Gupta P, Rahmani M, Zhang G, Hamed H, et al. Caspase-, cathepsin-, and PERK-dependent regulation of MDA-7/IL-24-induced cell killing in primary human glioma cells. Mol Cancer Ther. 2008; 7: 297-313.

42. Pataer A, Vorburger SA, Barber GN, Chada S, Mhashilkar AM, Zou-Yang H, et al. Adenoviral transfer of the melanoma differentiation-associated gene 7 (mda7) induces apoptosis of lung cancer cells via up-regulation of the double-stranded RNA-dependent protein kinase (PKR). Cancer Res. 2002; 62: 2239-43

43. Yacoub A, Mitchell C, Lebedeva IV, Sarkar D, Su ZZ, McKinstry R, et al. mda-7 (IL-24) Inhibits growth and enhances radiosensitivity of glioma cells in vitro via JNK signaling. Cancer Biol Ther. 2003; 2: 347-53.

44. Lebedeva IV, Sarkar D, Su ZZ, Kitada S, Dent P, Stein CA, et al. Bcl-2 and $\mathrm{Bcl}-\mathrm{x}(\mathrm{L})$ differentially protect human prostate cancer cells from induction of apoptosis by melanoma differentiation associated gene-7, mda-7/IL-24. Oncogene. 2003; 22: 8758-73.

45. Ramesh R, Mhashilkar AM, Tanaka F, Saito Y, Branch CD, Sieger K, et al. Melanoma differentiation-associated gene $7 /$ interleukin (IL)-24 is a novel ligand that regulates angiogenesis via the IL-22 receptor. Cancer Res. 2003; 63: 5105-13.

46. Su Z, Emdad L, Sauane M, Lebedeva IV, Sarkar D, Gupta P, et al. Unique aspects of mda-7/IL-24 antitumor bystander activity: establishing a role for secretion of MDA-7/IL-24 protein by normal cells. Oncogene. 2005; 24: 7552-66.

47. Miyahara R, Banerjee S, Kawano K, Efferson C, Tsuda N, Miyahara Y, et al. Melanoma differentiation-associated gene-7 (mda-7)/interleukin (IL)-24 induces anticancer immunity in a syngeneic murine model. Cancer Gene Ther. 2006; 13: 753-61.
48. Menezes ME, Bhatia S, Bhoopathi P, Das SK, Emdad L, Dasgupta S, et al. MDA-7/IL-24: multifunctional cancer killing cytokine. Adv Exp Med Biol. 2014; 818: 127-53. 